\title{
High-resolution infrared spectroscopy as a tool to detect false positives of transit search programs ${ }^{\star}$
}

\author{
E. W. Guenther ${ }^{1,2}$ and L. Tal-Or ${ }^{3}$ \\ 1 Thüringer Landessternwarte Tautenburg, Sternwarte 5, 07778 Tautenburg, Germany \\ e-mail: guenther@tls-tautenburg.de \\ 2 Instituto de Astrofísica de Canarias, C/Vía Láctea, s/n, 38205 La Laguna (Tenerife), Spain \\ ${ }^{3}$ School of Physics and Astronomy, Raymond and Beverly Sackler Faculty of Exact Sciences, Tel Aviv University, Tel Aviv, Israel
}

Received 22 October 2009 / Accepted 25 May 2010

\begin{abstract}
Context. Transit search programs such as CoRoT and Kepler now have the capability of detecting planets as small as the Earth. The detection of these planets however requires the removal of all false positives. Although many false positives can be identified by a detailed analysis of the light-curves (LCs), the detections of others require additional observations. An important source of false positives are faint eclipsing binaries within the point spread function (PSF) of the target star. For example, triple stars are an important source of false positives. Unfortunately, most of the methods previously applied have difficulties in detecting these objects.

Aims. We develop a new method that allows us to detect faint eclipsing binaries with a separation smaller than one arcsec from target stars. We thereby focus on binaries that mimic the transits of terrestrial planets. These binaries can be either at the same distance as the target star (triple stars), or at either larger, or smaller distances.

Methods. A close inspection of the problem indicates that these systems contain either late-type stars, or stars of high extinction. Thus, in both cases the binaries are brighter in the infrared than in the optical regime. We show how high resolution infrared (hereafter IR) spectroscopy can be used to remove these false positives.

Results. For the triple star case, we find that the brightness difference between a primary and an eclipsing secondary is about 9-10 mag in the visual but only about 4.5-5.9 mag in the $K$-band. In the next step, we demonstrate how the triple star hypothesis can be excluded by taking a high-resolution IR spectrum. Simulations of these systems show that the companions can be detected with a false-alarm probability of $\sim 2 \%$, if the spectrum has a signal-to-noise ratio $(S / N) \geq 100$. We subsequently show that high-resolution IR spectra also allows to detect most of the false positives caused by foreground or background binaries.

Conclusions. If high resolution IR spectroscopy is combined with photometric methods, virtually all false positives can be detected without radial velocity (RV) measurements. It is thus possible to confirm transiting terrestrial planets with a modest investment of observing time.
\end{abstract}

Key words. methods: observational - techniques: spectroscopic - planetary systems - binaries: eclipsing - infrared: stars

\section{Introduction}

Space-based programs such as CoRoT and Kepler searching for transiting planets now have the capability of detecting planets as small as one or a few Earth-radii (Leger et al. 2009; Queloz et al. 2009). An outstanding problem however, is the detection of false positives, i.e. objects that have light-curves resembling those of a transiting planet but which are something else. Excluding these false positives is an essential part of any transit search program, particularly those focusing on very small planets.

In a detailed study, Brown (2009) investigated the different possibilities for false-alarms, and concluded that diluted binaries are the dominant sources of false positives for space-based transit search programs. These can be either a foreground or background eclipsing binaries too close to the target to be resolved. Triple systems of small separation can be considered as a special case of a diluted binary. For the Kepler mission Gautier et al. (2007) and Caldwell et al. (2006) demonstrated that diluted binaries can be detected if they are separated from the target star by more than a quarter of a pixel, corresponding to about one arcsec on the sky.

* Partly based on observations obtained at the European Southern Observatory at Paranal, Chile in program 081.C-0033(A).
For the CoRoT mission, Almenara et al. (2009) showed that $83 \%$ of the transit candidates are identified as false positives by carefully analysing the light-curves. In the case of CoRoT, an important way to discriminate eclipsing triple systems from transiting planets is to use the colour difference in and out of transit (see also O'Donovan et al. 2006; Tingley 2004). This method was also used for CoRoT-7b but the $S / N$ ratio in the blue and green channel alone was found to be insufficient to exclude a late type M-star (Leger et al. 2009). This is because it is not only necessary to detect the transit in both colours but to measure the depth of the transit with sufficient accuracy. Furthermore the study by Almenara et al. (2009) show that another $5 \%$ of the transit candidates found by CoRoT are planets, and the remaining $12 \%$ are false positives that require additional observations.

The efficiency in removing false positives is so high because CoRoT performs three colour photometry. Follow-up observations thus concentrate on only $17 \%$ of the candidates, $30 \%$ of which are planets. The first step of the follow-up program is to identify possibly contaminating stars in the Exo-Dat database (Deleuil et al. 2009), and observe them in and out of transit (Deeg et al. 2009). Although these methods allow us to remove many false positives, some remain. 
The most important remaining case is a faint eclipsing binary with a separation of smaller than an arcsec from the target. These eclipsing binaries may be either in the background, the foreground, or at the same distance as the target star, as in a triple system.

In this article, we discuss a technique for identifying false positives by detecting the infrared CO lines of eclipsing binary $\mathrm{M}$ stars in the combined light of blended systems. This important, because methods based on RV-measurements require much observing time to detect planets. For example, 106 RV measurements were necessary to determine the mass of CoRoT-7b, a planet of $4.8 \pm 0.8 M_{\text {Earth }}$ and an orbital period of 0.8535 days (Queloz et al. 2009). In the case of a very low-mass planet in a long period orbit, it might be entirely impossible to confirm it by means of RV measurements. For example, the semi-amplitude of an earth-like planet orbiting at $1 \mathrm{AU}$ is only $0.09 \mathrm{~m} \mathrm{~s}^{-1}$. It is thus important to develop methods that allow to us verify transiting extrasolar planets without RV measurements. The method assumes that differences in RV - either from orbital motion in triple systems or simple line-of-sight differences - separate the spectral lines in wavelength space.

\section{Excluding triple systems}

In this section we describe how triple systems can be excluded as source of false positives by means of high-resolution infrared spectroscopy.

\subsection{The brightness difference between the primary and secondary is smaller in the IR}

As outlined above, triple systems may be a source of false positives but how relevant are they? Since the CoRoT and Kepler mission focus on main-sequence $\mathrm{F}, \mathrm{G}$, and $\mathrm{K}$ stars $\left(M_{*}=0.5\right.$ to $1.7 M_{\odot}$ ), only these stars are discussed in the following. According to Tokovinin (2008) at least $8 \%$ of the solar-type stars are in systems containing three or more stars. Dynamically stable triple systems have to be hierarchical with $P_{\mathrm{L}} / P_{\mathrm{S}}>5$ (Eggleton 2006). This means that even for an eclipsing system with an orbital period of one year, the separation between the primary and the eclipsing system can be as small as 3.0 AU. If we consider the canonical 12th magnitude G-star, this means that for the given semi-major axis, the ratio of the ratio of semi-major axis to distance can produce a maximum separation as small as 0.01 arcsec.

Using the results obtained by Tokovinin (2008), we find that the probability of a triple system containing two M-stars with an orbital period of one year is between 0.1 and $0.4 \%$. The probability of a triple system containing two eclipsing M-stars with an orbital period of up to one year is $\geq 10^{-4}$. Since the CoRoT and Kepler both survey $10^{5}$ stars, triple systems are an important source of false positives. It is thus unsurprising that CoRoT has already found at least one such system.

The positives aspect of a triple star is that all three components are at about the same distance from the Earth. Thus, given the depth of the transit, the spectral type of the primary, and the period of the transit, all relevant parameters of a hypothetical triple star system can be fixed. For example, an eclipsing binary that mimics a transiting planet such as the Earth would have to consist of two M-stars. The most interesting case is a transit that mimics a planet such as the Earth.

What would be the properties of a triple star causing such a LC? The triple star will consist of a F, G, K-star primary and eclipsing M-star binary. The two relevant cases for the M-star binary are:

- an eclipsing system consisting of two identical stars, such as YY Gem. In this case, the primary and secondary eclipse would have the same depth;

- a eclipsing system where the secondary eclipse is too shallow to be detected in the analysis of the LC. These are systems in which one component is much fainter than the other.

Unequal mass (M-star) binaries for which the secondary eclipse is detectable do not have to be considered here, as these false positives would already be removed in the analysis of the LC. There are two other less relevant cases. One is that $i, \Omega$, and $e$ are such that the primary eclipse is observed but not the secondary. We do not have to discuss this case separately, because the method applied to remove this case is identical to when one component is much fainter than the other. The method for excluding these two cases are identical.

Another possibility is a binary with an eccentric orbit in which the impact parameter is such that two transits of equal duration and depth are produced by stars of different radii. This case is very unlikely. Since the method for excluding this case is the same as for the case of identical stars, we do not have to discuss this case separately either. We thus have to exclude only the two relevant cases given above.

Transits of binaries can be either central or grazing. For a given transit depth, the eclipsing stars are brighter if the transit is grazing rather than central. Since binaries with central eclipses are more difficult to detect, we focus on central eclipses. In additional, all values are given for a transiting planet as the Earth but can be scaled to larger or smaller planets.

Since the depth of the transit is simply the squared of the ratio of the radii, the depth depends not only on the size of the planet but also the size of the star. The transit is shallower if the star has a larger diameter, and deeper if the star has a smaller diameter. The depth of the transit of a terrestrial planet is $5.8 \times$ $10^{-5}$ for an F5V-star, $9.6 \times 10^{-5}$ for a G5V-star, and $1.5 \times 10^{-4}$ for a $\mathrm{K} 5 \mathrm{~V}$ star.

What would be the properties of an eclipsing binary that mimics the transit of a terrestrial planet? In the case of an F5V star, the brightness-ratio of the primary to the secondary (the brighter of the two eclipsing stars) would be $I_{\text {sec }} / I_{\text {prim }} \leq 5.8 \times$ $10^{-5}$. This corresponds to a brightness-difference $m_{\mathrm{sec}}-m_{\text {prim }} \leq$ $10.6 \mathrm{mag}$ in the visual. Figure 1 shows the maximum difference in mag between primary and the secondary in the $V, J$, $H, K$-band $\left(\Delta m_{V}, \Delta m_{J}, \Delta m_{H}, \Delta m_{K}\right)$ for main-sequence stars of different spectral types. The brightness difference between the hypothetical companion and the primary depends on the spectral type of the primary star, because the depth of the transit depends on the size of the host star. The brightness difference is thus smaller for a main sequence K-star than for an F-star (again for a transit of a planet of the same size as the Earth).

At first glance, we may consider it straightforward to acquire a high-resolution spectrum in the optical regime with a sufficient $S / N$ to detect the companion. The spectral lines of the primary and the eclipsing binary would be well separated from both each other and the primary. For example, the semi-amplitude of an eclipsing binary consisting of two $0.09 M_{\odot}$-stars with an orbital period of one year is $18 \mathrm{~km} \mathrm{~s}^{-1}$. However, the problem is that the brightness difference in the optical is about $10 \mathrm{mag}$. To achieve a detection we would thus require a $S / N$ of 10000 . As we now show, the situation is more promising in the IR.

To estimate the brightness of the hypothetical eclipsing binary, we can assume that all three stars are at about the same 
E. W. Guenther and L. Tal-Or: High-resolution infrared spectroscopy as a tool to detect false positives

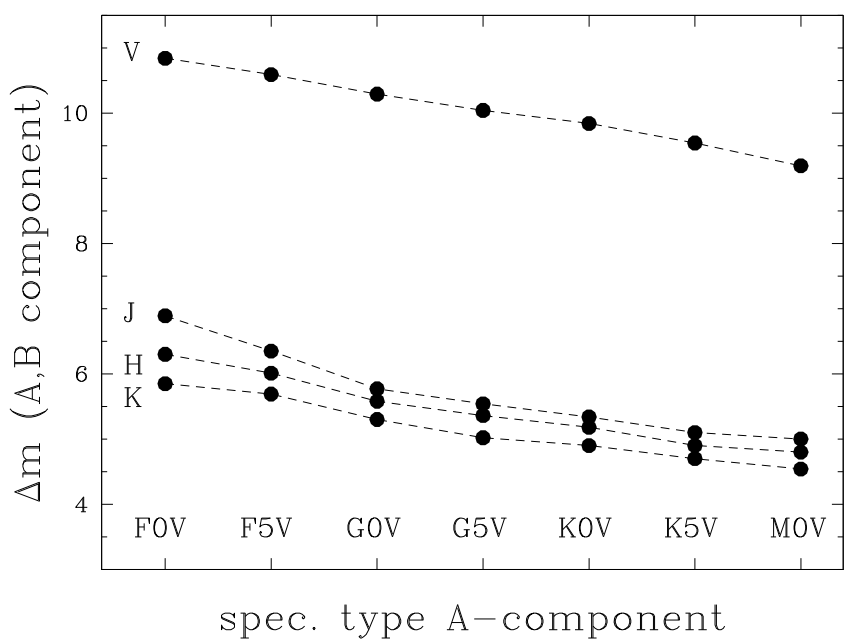

Fig. 1. Maximum difference in brightness between the $\mathrm{A}$ and the $\mathrm{B}$ component in mag $\left(\Delta m_{V}, \Delta m_{J}, \Delta m_{H}, \Delta\right)$.

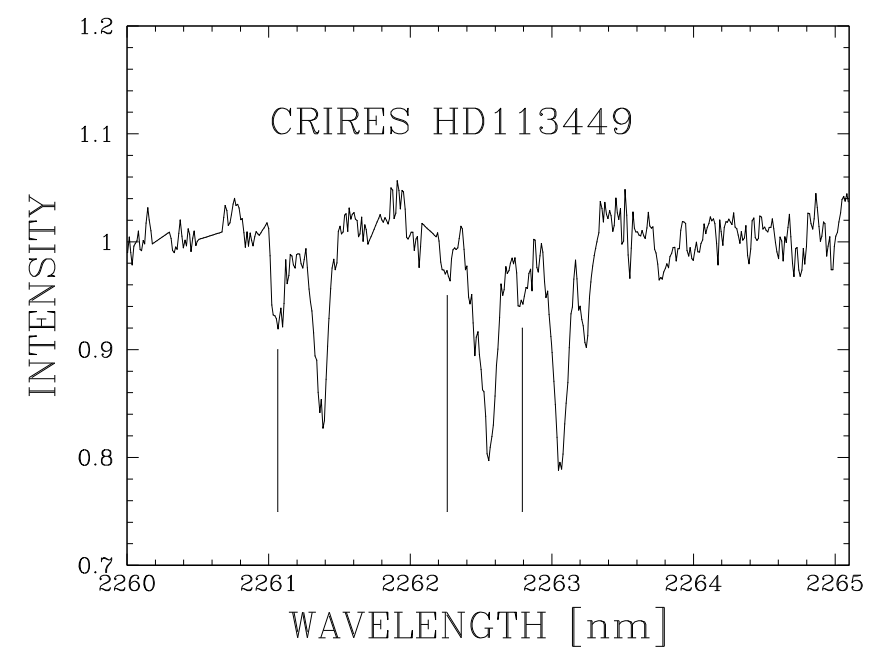

Fig. 2. CRIRES spectrum of the binary HD 113449 consisting of a G5V primary and an M3V secondary. Spectral lines of the secondary are marked.

distance from Earth. For a given brightness difference, we can thus calculate the spectral-type of the hypothetical companion. We find that the hypothetical companion would have a spectraltype in the range from M4V to M7V, if we were to assume a central transit. Using these hypothetical-companion spectral-types, we can derive the brightness differences for any other spectral region using the information given in Henry et al. (2006). For a grazing transit, the spectral-types would be earlier and the brightness difference correspondingly smaller.

As expected, the brightness difference between a mainsequence $\mathrm{F}, \mathrm{G}$, or $\mathrm{K}$-star and an $\mathrm{M}$-star is much smaller in the IR than in the optical regime. The brightness difference in the $K$-band is only $5.7 \pm 0.2 \mathrm{mag}$ for a main sequence F-star, $5.0 \pm 0.2 \mathrm{mag}$ for a G-star, and $4.8 \pm 0.2 \mathrm{mag}$ for a K-star, respectively (Fig. 1).

Since it has already been demonstrated that faint late-type secondaries of binaries can be efficiently detected by using highresolution IR spectroscopy, we can also use this method to detect false positives (Mazeh et al. 2009). A practical demonstration that an M-star companion of G-star can be detected is shown in Fig. 2. The figure shows a CRIRES spectrum HD 113449

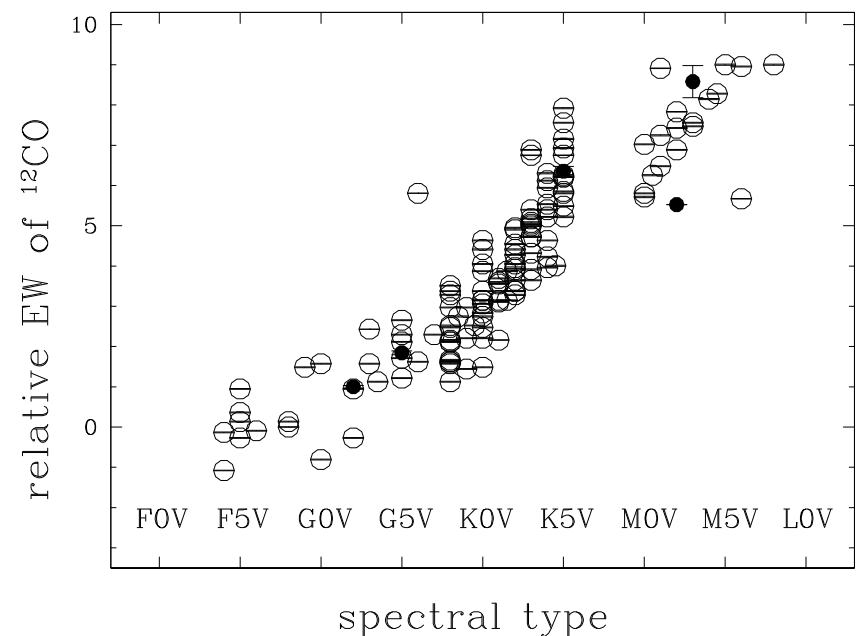

Fig. 3. Equivalent width of the ${ }^{12} \mathrm{CO}$ lines in the $K$-band relative to the strength of these lines in a G2V star.

consisting of a G5V primary and an M3V secondary. Spectral lines of both components are clearly visible.

\subsection{Using the CO-lines in the infrared}

In Sect. 2.1, we showed that the hypothetical companions are in the limiting cases M4V to M7V-stars, and pointed out that highresolution IR spectroscopy can be used to detect these false positives. We now discuss the instrumental requirements, illustrating how it works in practice.

The most effective way to exclude an M-star companion is to observe a region in the spectrum, in which the spectral lines of an M-star are stronger than the lines from an F, G, or K star. For example we consider that a spectral-line is absent in the primary but has a depth of $100 \%$ in the secondary, and that the secondary would be 5 mag fainter. In this case, a spectrum with a $S / N$ of 100 would be required to detect the secondary. If the line were only $10 \%$ deep, we would need a spectrum with a $S / N$ of 1000 . However, if the secondary exhibited 100 of these lines and we used the cross-correlation method to average them, we could still detect the secondary using a spectrum with a $S / N$ of 100 .

Because many high-resolution IR spectrographs have a rather limited wavelength coverage, we now discuss which part of the spectrum would be most suitable. For three reasons, the CO overtone lines (2.38 to $2.45 \mu \mathrm{m}$ ) seem to be the most suitable set of lines in the $J, H$, and $K$-bands:

- CO has a large number of deep lines ( $\sim 50$ lines);

- the overtone lines are in the $K$-band, where the brightnessdifference between an M-star and a G-star is smaller than in the $J$ or $H$-band;

- the lines are much stronger for an M-star than for an F, G, or K-type star (Fig. 3).

Goorvitch (1994) provides a list of $50{ }^{12} \mathrm{C}^{16} \mathrm{O}$ lines in the $K$-band. The atlas published by Wallace et al. (2001) shows that these lines are typically 50\% deep in a sun-spot (corresponding to an M3V star).

The third reason given above requires some additional discussion. The optimal way of quantifying the strength of the lines would be to use high-resolution spectra of stars in the spectral-type range between early $\mathrm{F}$ and late $\mathrm{M}$. High-resolution spectra of the Sun, (G2V), 61 Cyg B (K7V), and a sunspot (M3V) are shown in Fig. 5 (Livingston \& Wallace 1991; 
Wallace \& Hinkle 1996; Wallace et al. 2001). Unfortunately, until now there has been an insufficient number of high-resolution spectra to quantify the line depth for all spectra types but sufficient low- and medium- resolution spectra. The strength of the lines is often expressed in terms of the ${ }^{12} \mathrm{CO}(2,0)$-bandhead index. For example, Mármol-Queraltó et al. (2008) developed a new CO-index that is less dependent on the resolution of the spectrograph. This index can thus be used to quantify the strength of the lines using low and medium resolution spectra. To calibrate this CO-index, all we have to do is to measure the line depth in a few very high resolution spectra. For this purpose, we use FTS spectra published by Wallace et al. (1996), and scale the $\mathrm{CO}$-index values accordingly. Figure 3 shows the strength of the ${ }^{12} \mathrm{CO}$-lines for different spectral types relative to the sun.

In good agreement with the older data (Kleinmann \& Hall 1986), the new measurements show that the strengths of the CO-lines increase for stars with spectral types up to M6V. For later spectral types, the strengths decrease again (Cushing et al. 2000), but this is not relevant to the case of the triple system. We discuss this case in the case of a foreground binary. Figure 6 shows the appearance of the cross-correlation function for companions that are 3, 4, 4.5, and 5 mag fainter, and shifted by $13 \mathrm{~km} \mathrm{~s}^{-1}$.

We now derive the equation for the $S / N$ required to exclude a binary mimicking a planet of a given radius. We assume that there are at least $50 \mathrm{CO}$-lines within the spectral range observed, and that the resolution is high enough to resolve them $(\lambda / \Delta \lambda \geq$ $30000)$. The $S / N$ that is required is

$$
S / N \geq 100 \cdot 2.512^{\left(5-\Delta m_{K}\right)} \cdot \frac{8}{\operatorname{RelCO}} \cdot \frac{R_{\text {Earth }}}{R_{\text {planet }}},
$$

where $\Delta m_{K}$ is the brightness difference between the primary and secondary, $\Delta m_{K}$ is 5.9-4.5 mag for F0V to M0V stars (Fig. 1), $R_{\text {planet }}$ is the radius of the planet that is to be confirmed, and RelCO is the relative strength of the CO-line shown in Fig. 3 (RelCO $\sim 8$ for M-stars). Using this equation, we conclude that a $S / N \sim 100$ is sufficient for identifying this kind of false positive.

With TODCOR we now demonstrate that the detection is possible. TODCOR is a 2-dimensional cross-correlation method that has already been indeed applied to detect very faint companions in IR spectra (Mazeh et al. 2009). We performed simulations in which we used the FTS spectra (Livingston \& Wallace 1991; Wallace \& Hinkle 1996) and resampled them to a resolution of $\lambda / \Delta \lambda=60000$ to mimic CRIRES observations with a 0.4 arcsec slit. Our simulated binary consisted of a G2V primary and an M-star companion, which was shifted by $20 \mathrm{~km} \mathrm{~s}^{-1}$, was 5 mag fainter in the $K$-band. We finally added random noise to the spectra and carried out 1300 simulations with different $S / N$-ratios. For a $S / N$-ratio of 100 , we performed 200 simulations. Given that there are three outliers in all our simulations, the false-alarm probability is $\sim 2 \%$ for a $S / N$ of 100 . These simulations thus confirm our expectation. Figure 4 shows the RV measurements obtained with TODCOR for different values of $S / N$. For higher $S / N$ the false-alarm probability is lower, but increases rapidly towards lower $S / N$. For example, at a noise-level of 85 , thefalse-alarm probability is already $5 \%$. To exclude falsealarms of terrestrial planets, we thus recommend using spectra with a $S / N \geq 100$.

In principle, it could be argued that the fundamental bands are more suitable than the overtone-bands, since they are about a factor six stronger (Heras et al. 2002). However, these lines are in the wavelength range between 4.30 and $4.70 \mu \mathrm{m}$, thus almost unobservable from the ground.

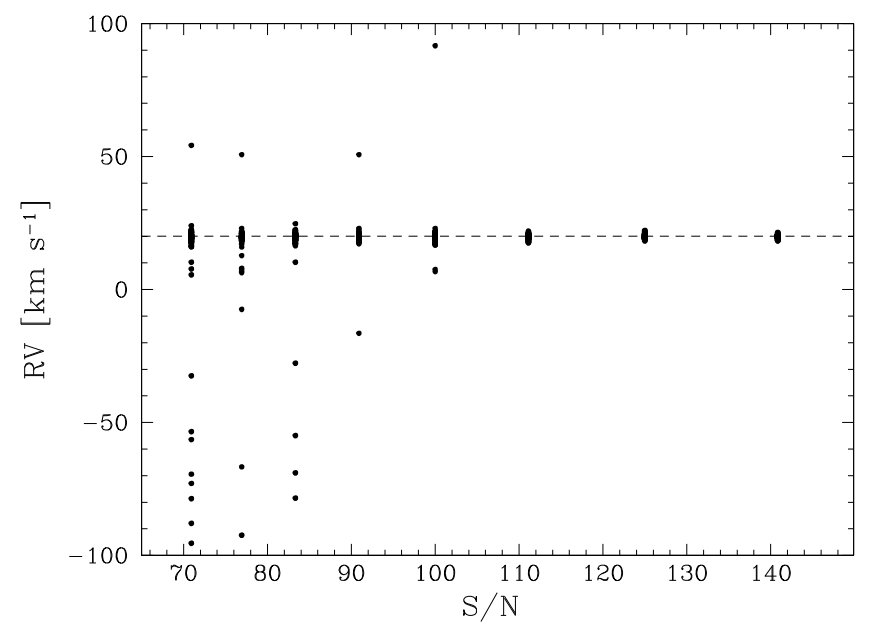

Fig. 4. RV-measurements obtained with TODCOR for a simulated binary consisting of a G2V primary and an M-star secondary that is 5 mag fainter in the $K$-band for different $S / N$-ratios.

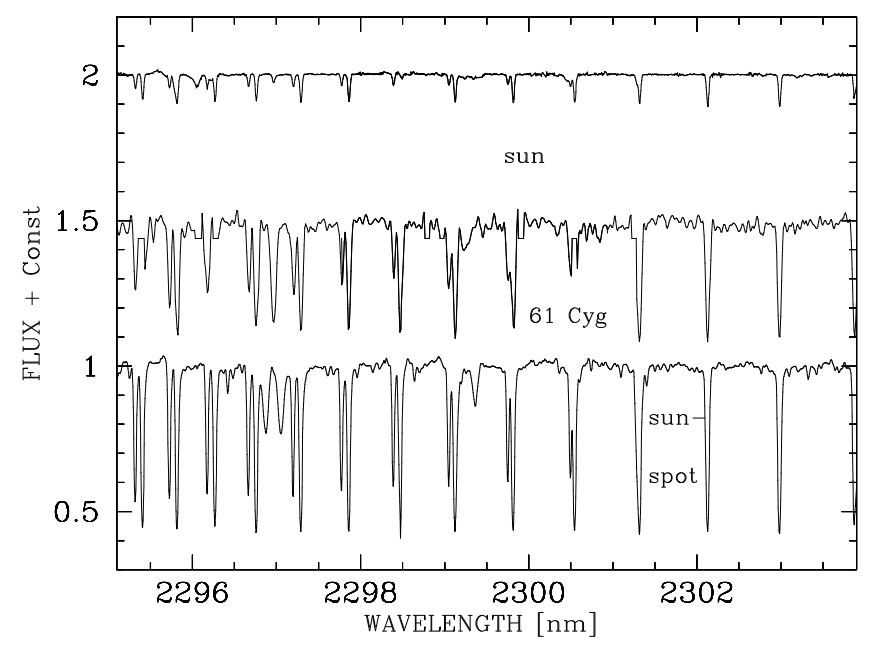

Fig. 5. Spectra of the Sun $(\mathrm{G} 2 \mathrm{~V}), 61 \mathrm{Cyg}$ B (K7V), and a sunspot $(\mathrm{M} 3 \mathrm{~V})$, demonstrating the increase in the strength of the CO-lines towards later spectral types.

\section{Excluding a binary in the background}

Another possibility to be excluded is that there is an unrelated eclipsing binary in the background. Although most of the background binaries can be removed by analysing the light-curves not all them can be removed in this way. The usual methods have problems in removing binaries that are faint and close to the primary. In the following, we show that high-resolution IR spectroscopy also helps to remove these false positives. As before, the brightness difference can be as large as 9.2-10.8 mag in the optical regime (Fig. 1). Would the brightness difference be smaller in the IR? Calculating the brightness of the background star in the IR is not easy, because we do not know its spectral type. We thus have to consider two cases:

- at least one of the stars is a giant star;

- the eclipsing background binary consists of two mainsequence stars.

Since typical targets are between 10th and 14th magnitude, we have to exclude there being background binaries as faint as 19th or even 25 th mag in the visual. 
E. W. Guenther and L. Tal-Or: High-resolution infrared spectroscopy as a tool to detect false positives

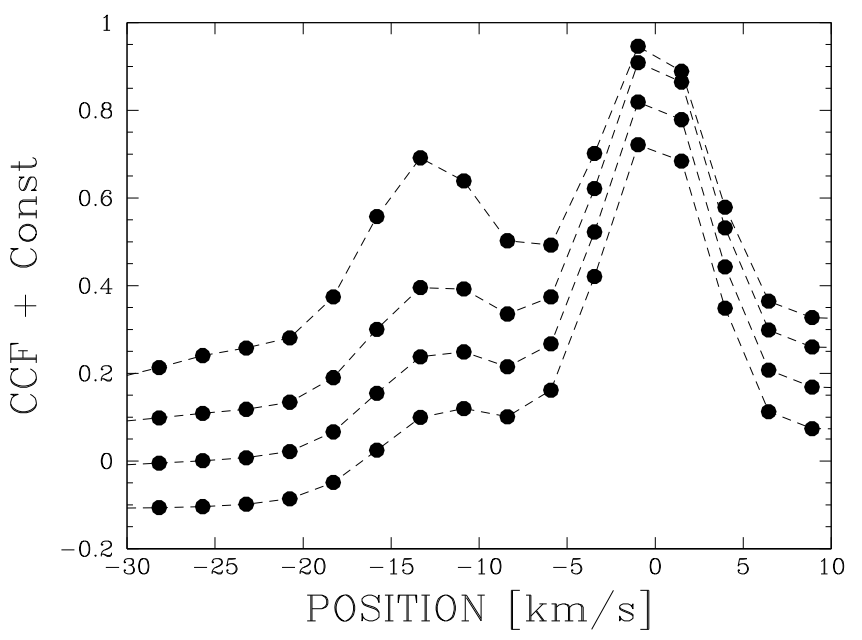

Fig. 6. Cross-correlation function for a solar-like star and an M-star companion which is $3,4,4.5$ and 5.0 mag fainter.

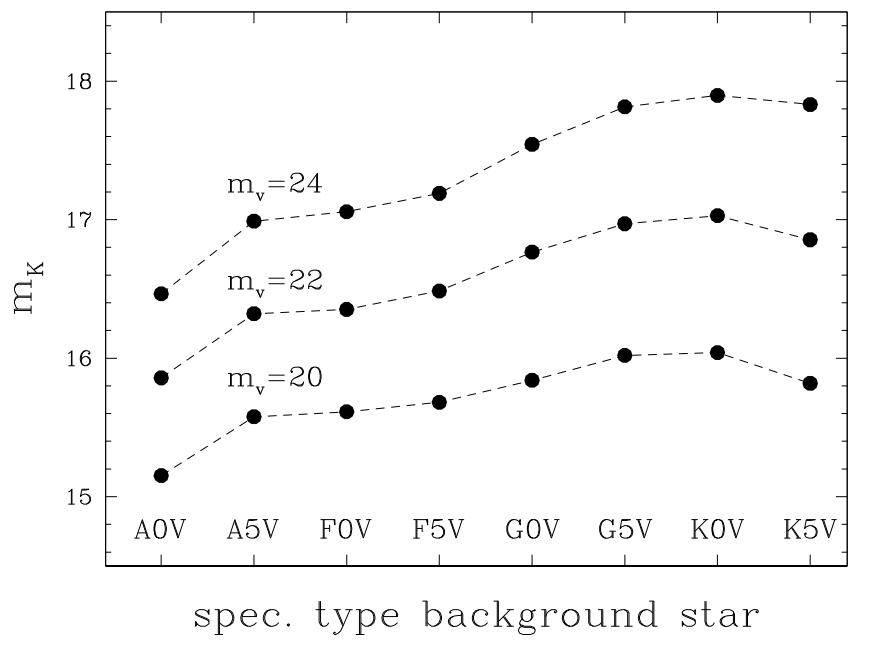

Fig. 7. Brightness of an unrelated main-sequence background star with $m_{V}=20,22$, and 24 in the $K$-band. Because the extinction is smaller in the IR, the objects are much brighter in this wavelength regime.

Giant stars in the field would certainly be much brighter in the IR than in the optical regime. As shown in Deleuil et al. (2009), about $70 \%$ of the giant stars in the CoRoT fields are $\mathrm{K}$-stars, the remainder being G-type giants. Without extinction, the $V-K$ colours are in the range between 2.3 and 3.6 mag for K-giants, and 1.8 and 2.2 mag for G-giants, respectively. If we take into account that the surveys are carried out close to the plane of the Milky Way, the extinction will make these objects even redder. Brown (2009) finds that the $J-K$ colours of background giant stars are between 0.5 and 1.5 mag. Background giants are thus at least two mag brighter in the $K$-band than in the optical regime. Thus, acquiring IR spectra can greatly increase our likelihood of detecting background giants. This is especially true for K-giants, since $\mathrm{K}$-stars have strong CO-line overtone lines.

Dust extinction also changes the colours of main-sequence stars. Figure 7 shows the brightness of background stars in the $K$-band. To estimate the interstellar extinction, we used a model published by Beatty \& Gaudi (2008) and assumed that the observation was carried out in the Galactic plane. Because the extinction is much smaller in the $K$-band than in the visual, background stars are much brighter in the IR than in the optical regime. Our estimates are confirmed by the results of Deleuil et al. (2009), who show that faint stars in the CoRoT fields have red colours.

Examples of background binaries that cannot be excluded using IR spectroscopy are sdBs and cataclysmic variables because these objects are blue. While cataclysmic variables cannot have orbital periods of a year, this is possible for sdBs and white dwarf binaries. Maxted et al. (2001) give a space density for sdBs of $10^{-6} \mathrm{pc}^{-3}$. The space density of sdBs is thus very low, and this case is unlikely. If we were to take into account that a sdB can only have blue colours if the extinction is low, the binary would have a distance of about $100 \mathrm{kpc}$ to be of $m_{V}=24$. Since we observe in the plane of the Milky Way, this case is not possible. Thus, neither sdBs nor CVs can cause false positives.

In addition to spectroscopy, IR photometry can be used to exclude unrelated background binaries. If we find that the transit is deeper in the IR, we can infer that it is a binary.

\section{Excluding a binary in the foreground}

We finally discuss the possibility of an unrelated eclipsing binary in the foreground. As for the background objects, the brightness difference can be as great as $10.8 \mathrm{mag}$ in the visual. A binary in the foreground that is 10.8 mag fainter has a spectral type later than M6V, unless it consists of white dwarfs, in which case it could be a M, L, T type binary. This object would again be much brighter in the IR than in the optical. To demonstrate this, we assume a binary consisting of two objects such as 2M150716 , which has a spectral type L5V, and brightnesses of $m_{V}=$ $22.9 \pm 0.5, m_{K}=11.4 \pm 0.2$ (Dahn et al. 2002). This means that a binary consisting of two of these objects would be as bright as the primary in the IR! It would certainly be very easy to exclude this case. At M9.5, $V-K$ is 9.3 mag, making the detection of a foreground binary in the IR easier than either a triple system or a background binary.

We can not exclude using IR spectroscopy the presence of white dwarf binary. Maxted et al. (2001) calculate a space density of white dwarf binaries of $5 \times 10^{-3} \mathrm{pc}^{-3}$. The probability of finding an unrelated eclipsing white dwarf system with an orbital period of about a year within one arcsec from the target star is $<10^{8}$ and thus irrelevant.

\section{Conclusions}

Most of the methods that have been used to detect false-positives in transit search programs have difficulties in detecting faint eclipsing binaries within the PSF of the target star. Examples of these binaries triple stars containing two eclipsing M-stars. The problem is that the separations of the stars in these systems can be too small to be spatially resolved. Since triple stars are common, they are an important source of false positives. Another source being eclipsing background binaries. We have demonstrated that both types of false positives can be effectively eliminated using high resolution IR spectroscopy. The reason for this is that eclipsing stars either are at large distances and thus suffer from extinction, or are nearby late-type stars. Thus, in both cases, they are brighter in the IR than in the optical regime. If high resolution IR spectroscopy is combined with photometric methods, virtually all false positives can be detected without RV measurements. It is thus possible to confirm transiting planets with a modest investment of observing time.

Acknowledgements. E.G. would like to thank Stephan Geier for his information about sdBs and CVs. 


\section{References}

Almenara, J. M., Deeg, H. J., Aigrain, S., et al. 2009, A\&A, 506, 337

Beatty, T. G., \& Gaudi, B. S. 2008, ApJ, 686, 1302

Brown, T. M. 2003, ApJ, 593, L125

Caldwell, D. A., Batalha, N. M., Borucki, W. J., et al. 2006, BAAS, 38, 1189

Cushing, M. C., Rayner, J. T., \& Vacca, W. D. 2005, ApJ, 623, 1115

Dahn, C. C., Harris, H. C., Vrba, F. J., et al. 2002, AJ, 124, 1170

Deeg, H. J., Gillon, M., Shporer, A., Rouan, D., \& Stecklum, B. 2009, A\&A, 506,343

Deleuil, M., Moutou, C., Deeg, H. J., et al. 2006, ESA SP, 1306, 341

Deleuil, M., Meunier, J. C., Moutou, C., et al. 2009, AJ, 138, 649

Deming, D., \& Plymate, C. 1994, ApJ, 426, 382

Eggleton, P. 2006, Evolutionary Processes in Binary and Multiple Stars, ed. P. Eggleton (Cambridge, UK: Cambridge University Press)

Gautier, T. N., Borucki, W. J., Caldwell, D. A., \& Koch, D. G. 2007, Transiting Extrasolar Planets Workshop, 366, 219

Goorvitch, D. 1994, ApJS, 95, 535
Henry, T. J., Jao, W.-C., Subasavage, J. P., et al. 2006, AJ, 132, 2360

Heras, A. M., Shipman, R. F., Price, S. D., et al. 2002, A\&A, 394, 539

Kleinmann, S. G., \& Hall, D. N. B. 1986, ApJS, 62, 501

Leger, A., Rouan, D., Schneider, J., et al. 2009, A\&A, 506, 287

Livingston, W., \& Wallace, L. 1991, NSO Technical Report, Tucson: National Solar Observatory, National Optical Astronomy Observatory

Mazeh, T., Prato, L., Simon, M., et al. 2002, ApJ, 564, 1007

Mármol-Queraltó, E., Cardiel, N., Cenarro, A. J., et al. 2008, A\&A, 489, 885

Maxted, P., Marsh, T. R., \& North, R. C. 2001, 12th European Workshop on White Dwarfs, 226, 187

O’Donovan, F. T., Charbonneau, D., Torres, G., et al. 2006, ApJ, 644, 1237

Queloz, D., Bouchy, F., Moutou, C., et al. 2009, A\&A, 506, 303

Tokovinin, A. 2008, MNRAS, 389, 925

Tingley, B. 2004, A\&A, 425, 1125

Wallace, L., \& Hinkle, K. 1996, ApJS, 107, 312

Wallace, L., Livingston, W., Hinkle, K., \& Bernath, P. 1996, ApJS, 106, 165

Wallace, L., Hinkle, K., \& Livingston, W. C. 2001, NSO Technical Report \#01001, Tucson: National Solar Observatory 